\section{A evitabilidade de óbitos entre idosos em São Paulo, Brasil: análise das principais causas de morte}

\author{
Avoidability of deaths from chronic illnesses in \\ elderly individuals in São Paulo, Brazil
}

\author{
La evitabilidad de óbitos entre ancianos en \\ São Paulo, Brasil: análisis de las principales \\ causas de muerte
}

Solange Kanso 1,2 Dalia Elena Romero 3 Iuri da Costa Leite 4 Aline Marques 3

\footnotetext{
${ }^{1}$ Instituto de Pesquisa Econômica Aplicada, Rio de Janeiro, Brasil. 2 Ibmec, Rio de Janeiro, Brasil.

3 Instituto de Comunicação e Informação Cientifica e Tecnológica em Saúde Fundação Oswaldo Cruz, Rio de Janeiro, Brasil. ${ }^{4}$ Escola Nacional de Saúde Pública Sergio Arouca, Fundação Oswaldo Cruz, Rio de Janeiro, Brasil.

Correspondência S. Kanso

Instituto de Pesquisa Econômica Aplicada. Av. Presidente Antônio Carlo 51, 14o andar, Rio de Janeiro, RJ 20020-010, Brasil. solange.kanso@gmail.com
}

\begin{abstract}
Avoidable causes of death have become an important indicator for evaluating health services. Such deaths would not occur if there were adequate prevention, early diagnosis, and treatment and the adoption of appropriate technologies. This article analyzes the pattern and magnitude of avoidable causes of death for the principal causes of death among elderly individuals up to 74 years of age, by gender, in São Paulo State, Brazil. The study further estimated the impact of avoidable causes of death on life expectancy among the elderly, and $62.5 \%$ of the deaths among elderly up to 74 years in São Paulo were due to these causes. The principal avoidable causes of death constituted $82.6 \%$ of all avoidable deaths, featuring hypertensions and tobacco-associated tumors. Life expectancy at 60 would increase by $20 \%$ if these deaths were avoided. Male excess mortality was observed in nearly all of the avoidable causes of death analyzed here, which could be related to greater exposure to risk factors and less use of health services by men. Prevention and health promotion measures should take these sharp gender differences into account.
\end{abstract}

Cause of Death; Chronic Disease; Aged; Health Public Policy

\section{Resumo}

As causas de morte evitáveis tornaram-se um importante indicador para avaliar os serviços de saúde. São óbitos que não deveriam ocorrer diante de adequadas ações de prevenção, tratamento, diagnóstico precoce e adoção de tecnologias apropriadas. Neste artigo, analisam-se o padrão e a magnitude das causas de morte evitáveis das principais causas de morte entre idosos com até 74 anos, por sexo, no Estado de São Paulo, Brasil. Estimou-se, ainda, o impacto das causas de morte evitáveis sobre a expectativa de vida dos idosos e observou-se que 62,5\% dos óbitos entre idosos com até 74 anos, em São Paulo, ocorreram por essas causas. As principais causas de morte evitáveis constituíram $82,6 \%$ do total de óbitos evitáveis, destacando-se a hipertensão e os tumores associados ao tabagismo. A expectativa de vida aos 60 anos aumentaria em 20\% se esses óbitos não ocorressem. A sobremortalidade masculina foi observada em quase todas as causas de morte evitáveis analisadas, o que pode estar relacionado à maior exposição a fatores de risco e à menor utilização dos serviços de saúde pelos homens. Recomenda-se que as ações de prevenção e promoção de saúde considerem as acentuadas diferenças de sexo e gênero.

Causas de Morte; Doença Crônica; Idoso; Políticas Públicas de Saúde 


\section{Introdução}

O rápido processo de envelhecimento da população mundial trouxe à tona um profícuo debate a respeito do alcance que a tecnologia moderna pode ter sobre a saúde dos idosos, no sentido de prolongar a vida e reduzir os níveis de incapacidade funcional 1 .

Desde 1970, o principal fator responsável pelo contínuo ganho de expectativa de vida nos países industrializados tem sido a redução nas taxas de mortalidade entre os idosos, especialmente nas taxas de mortalidade por doenças cardiovasculares e câncer. O declínio nessas taxas resulta de uma variedade de fatores, incluindo programas de promoção à saúde e intervenções médicas bem-sucedidas 2 .

Intrínseco ao processo de prolongamento da vida está o conceito de causas de morte evitáveis, fortemente associado à definição de mortalidade prematura, introduzida por Dempsey ${ }^{3}$, precursor do estudo realizado por Rutstein et al. ${ }^{4}$. A metodologia de causas de morte evitáveis foca sua atenção em um conjunto de doenças e agravos que poderiam ser eliminados, ou cujos efeitos poderiam ser controlados, por meio de ações de prevenção, diagnóstico e tratamento precoce, além de adoção de tecnologias mais apropriadas $4,5,6,7$, tornando-se importante indicador sentinela para orientar o processo de avaliação dos serviços de saúde e, assim, sinalizar falhas no sistema de atendimento 4,5 .

A classificação da evitabilidade de uma causa de morte, assim como as medidas e políticas de redução desta, depende dos conhecimentos e avanços da medicina, do desenvolvimento de novas tecnologias e de abordagens da saúde pública 8,9. A maioria dos estudos de mortalidade evitável foi realizada nas décadas de 1970 e 1980, principalmente com base em versões abreviadas da lista de causas definidas por Rutstein et al. 4 , ou em adaptações da lista de Charlton et al. 10 . Esta última reflete o consenso sobre o que poderia ser alcançável pelo atendimento médico e recursos disponíveis naquele momento. No entanto, desde então, tem havido avanços substanciais tanto no âmbito da qualidade dos cuidados de saúde, quanto no conhecimento médico 10 Em uma atualização de seu trabalho, Mackenbach 11, considerando os avanços na assistência médica para o tratamento de doenças isquêmicas do coração e câncer do reto, incluiu essas causas na classificação de evitabilidade.

Além dos avanços na tecnologia médica, a região geográfica aparece como um fator importante no processo de classificação de uma causa como sendo evitável. Por exemplo, a meningite e o diabetes mellitus foram incluídos no estudo realizado na Nova Zelândia 12 , mas a hipertensão e a asma foram excluídas das causas de morte evitáveis na Groenlândia ${ }^{13}$. Nesse sentido, o conceito vem sendo continuamente modificado, indo além das doenças transmissíveis, incluindo, de forma gradual, um conjunto de doenças crônico-degenerativas. 9,10,11,14,15,16,17.

Assim, o aumento da esperança de vida, principalmente no segmento populacional de idosos, e as evidências epidemiológicas sobre o caráter evitável das mortes prematuras por doenças crônicas, seja pela promoção de hábitos saudáveis, reduzindo o consumo do fumo e do álcool 18, seja pela prevenção da gravidade da doença, por meio do controle regular 19 , seja pelos avanços na tecnologia e no conhecimento médico ${ }^{1}$, constituem importantes argumentos para o uso do conceito de evitabilidade na análise da mortalidade da população idosa.

Em estudo recente, Gómez-Arias et al. 17, considerando a proposta original de Rutstein et al. ${ }^{4}$ e as modificações de Taucher 7 e de Holland 14, ampliaram a participação das doenças crônicodegenerativas na lista de causas de morte evitáveis, assim como as medidas associadas para sua redução, na Colômbia e em países com condições epidemiológicas e sanitárias similares.

Não existe consenso na literatura quanto à idade limite da vida 20,21, tampouco até que idade uma morte pode ser considerada evitável. O limite proposto inicialmente por Rutstein et al. ${ }^{4}$ foi de 65 anos, mas essa idade foi se ampliando progressivamente como resultado do aumento da expectativa de vida 22 . Em 2001, Tobias \& Jackson 23, com base em informações sobre a mortalidade da Nova Zelândia, estabeleceram 75 anos como limite. Nolte \& McKee 9 também assumiram esse limite, que, apesar de arbitrário, justifica-se, pois considera que a multicausalidade e a multiplicidade de fatores envolvidos na mortalidade das pessoas idosas dificultam a determinação da causa básica da mortalidade e também as chances efetivas de se evitar a morte após os 75 anos 24,25,26.

Em 2007, um grupo de especialistas elaborou a primeira Lista Brasileira de Causas Evitáveis para os menores de cinco anos e para aqueles indivíduos com idades entre 5 e 74 anos. ${ }^{27}$.

Entre 1991 e 2008, o Brasil experimentou um ganho de quase três anos na esperança de vida aos 60 anos de idade, que passou de 18,7 anos para 21,3 anos. Apesar desses avanços, a esperança de vida dos idosos brasileiros é menor do que a observada em países vizinhos, tais como Chile (22,3 anos) e Venezuela (22,0 anos), e menor ainda quando comparada à de países desenvolvidos, como a Suíça (24,7 anos) e o Japão (25,6 anos). Essa menor expectativa de vida observada 
entre os idosos no Brasil indica uma possibilidade real de redução da mortalidade nesse segmento populacional.

Há fortes evidências sobre a necessidade de uma abordagem de gênero nas condições de saúde e de morte durante o processo de envelhecimento. As mulheres têm maior prevalência de doenças não fatais, como artrites, sinusites e doenças do aparelho digestivo, enquanto os homens apresentam prevalência mais elevada por patologias fatais, como as doenças isquêmicas do coração e o enfisema pulmonar 28. Dessa forma, as mulheres vivem em média sete anos a mais do que os homens, tanto nos países desenvolvidos, quanto naqueles em desenvolvimento 29 .

O objetivo principal deste trabalho é analisar o padrão e a magnitude dos óbitos por causas de morte evitáveis, com ênfase nas principais causas entre idosos com até 75 anos, no Estado de São Paulo, Brasil, segundo o sexo. Pretendese, ainda, conhecer os ganhos potenciais na esperança de vida aos 60 anos, caso esses óbitos não ocorressem. A escolha de São Paulo se deve ao fato de o estado ter um registro de óbitos de boa qualidade: cobertura próxima de $100 \% 30$ e baixa proporção de causas maldefinidas $(6,6 \%)$ e inespecíficas $(9,1 \%) 31$. Outro critério utilizado na seleção refere-se ao fato de São Paulo registrar o maior índice de envelhecimento do país, aproximadamente 43 idosos para cada 100 pessoas menores do que 15 anos.

\section{Material e métodos}

A fonte de dados utilizada foi o Sistema de Informações sobre Mortalidade (SIM) do Ministério da Saúde, referente aos óbitos classificados como evitáveis ocorridos entre idosos, com idade entre 60 e 74 anos, residentes no Estado de São Paulo, em 2007. Para o cálculo das taxas, foram empregadas as estimativas da população de São Paulo, segundo sexo e idade, elaboradas pelo Instituto Brasileiro de Geografia e Estatística (IBGE) para o mesmo ano.

A análise foi dividida em três partes. Na primeira contemplaram-se os capítulos de causas de mortalidade com maior incidência de óbitos elencados no estudo de Gómez-Arias et al. 17, incluindo as causas maldefinidas. Na segunda, analisaram-se as principais causas de morte evitáveis observadas nesses capítulos. Por fim, foram calculados os ganhos na expectativa de vida aos 60 anos, excluindo-se os óbitos por causas evitáveis. Para análise dos dados, empregou-se o programa estatístico SPSS 15.0 para Windows (SPSS Inc., Chicago, Estados Unidos) e, para codificação das causas de óbitos, utilizou-se a 10ạ revisão da Classificação Internacional de Doenças (CID-10) 32 .

Foram construídos os seguintes indicadores por sexo: (1) mortalidade proporcional por causa - quociente entre os óbitos de idosos de 60 a 74 anos em decorrência de causa de morte (capítulo) e total de óbitos de idosos de 60 a 74 anos multiplicado por 100; (2) proporção de óbitos evitáveis - quociente entre os óbitos evitáveis (distribuídos nos capítulos) de idosos de 60 a 74 anos e o total de óbitos de idosos de 60 a 74 anos multiplicado por 100; (3) taxas de mortalidade por causa - quociente entre os óbitos de idosos de 60 a 74 anos em decorrência de causa de morte (capítulo) e a população de idosos de 60 a 74 anos multiplicada por $10 \mathrm{mil}$; (4) taxas de mortalidade por óbitos evitáveis - quociente entre os óbitos evitáveis (distribuídos nos capítulos) de idosos de 60 a 74 anos e a população de idosos de 60 a 74 anos multiplicada por 10 mil; (5) razão de taxas óbitos totais - quociente entre as taxas de mortalidade de óbitos totais entre homens e mulheres por causas de morte; (6) razão de taxas de óbitos evitáveis - quociente entre as taxas de mortalidade por óbitos evitáveis entre homens e mulheres por causas de morte.

\section{Classificação de causas de morte evitáveis}

Adotou-se a classificação de causas de morte evitáveis desenvolvida por Gómez-Arias et al. 17, pois esta incorpora as causas de morte de maior prevalência entre idosos. As causas de morte evitáveis foram classificadas em quatro grupos, segundo medidas de intervenção (Tabela 1):

- Prevenção primária: óbitos evitáveis por vacinação ou tratamento preventivo, como imunizações, vigilância epidemiológica, educação para a saúde e proteção específica para indivíduos que residem em áreas com deficiência na cobertura do saneamento básico.

- Atenção médica: óbitos evitáveis por diagnóstico e tratamento precoce, evitáveis pelos serviços médicos.

- Saneamento ambiental: óbitos evitáveis por ações sobre o meio ambiente, visando à prevenção e erradicação de fatores de riscos ambientais.

- Medidas mistas: óbitos evitáveis por meio da aplicação de medidas mais complexas, combinando serviços médicos e ações socioeconômicas relacionadas com as condições de desenvolvimento social. Subdividem-se em: MM1 - infecções do trato respiratório; MM2 - problemas maternos durante a gestação, o parto e o puerpério; MM3 - doenças da infância; MM4 - mortes violentas; MM5 - tuberculose; MM6 - problemas de deficiências nutricionais; MM7 - problemas 
Tabela 1

Causas de morte evitáveis segundo medidas de intervenção e subgrupos, entre idosos de 60 a 74 anos.

Medidas de intervenção e causas de morte

Código da CID-10

Prevenção primária (vacinação ou tratamento precoce)

Todas as zoonoses bacterianas (praga, tularemia, antraz, brucelose, mormo, amiloidose, febre de mordida de rato, entre outras)

Lepra e outras doenças por microbactérias

Difteria

Angina estreptocócica e escarlatina

Erisipela

Tétanos

Sífilis e outras doenças venéreas

Febre reumática aguda

Doenças reumáticas crônicas do coração

Infecção meningocócica

Febre amarela

Raiva

Sequelas de poliomielite

Hepatite B

Sequelas da lepra

Atenção médica (diagnóstico e tratamento precoce)

Tumor maligno da mama

Tumor maligno do útero

Tumor maligno da próstata

A20-A28

A30-A31

A36

A38

A46

A33-A34

A50-A63

100-102

105-109

A39

A95

A82

B91

B160, B161, B180, B181

B92

Diabetes mellitus

Doença hipertensiva

Bronquite, asma, enfisema e outras doenças das vias aéreas inferiores

C50

Doenças da cavidade oral, glândulas salivares e maxilares

C53-C55

C61

E10-E14

I10-I15

J40-J47

$\mathrm{K} 00-\mathrm{K} 14$

Outras doenças além do câncer de esôfago

K20-K23

úlcera gástrica ou duodenal

$\mathrm{K} 25-\mathrm{k} 28$

Gastrite e duodenite

$\mathrm{k} 29-\mathrm{k} 30$

Doenças do apêndice

K35-K38

Hérnia abdominal e obstrução intestinal

Doença diverticular do intestino

K40-K46, K56

Distúrbios gastrintestinais funcionais não classificadas em outra parte

K57

Colelitíase e outras desordens na vesícula

K59

K80-K83

K65

Peritonite

K75-K77

Abscesso hepático e sequelas de fígado tóxico

N17-N19

nsuficiência renal

590 *

nfecções renais

A40-A41

Outros transtornos dos órgãos genitais femininos

N80-N83

Doenças cerebrovasculares

Doenças isquêmicas do coração

160-169

120-125

Doença da válvula mitral

134

Insuficiência cardíaca congestiva

150

Tromboflebite

Litíase renal e ureteral

182

N20-N21

Cistite

Doenças dos órgãos genitais

N40-N51

Transtornos da mama

N60-N64

(continua) 
Tabela 1 (continuação)

Medidas de intervenção e causas de morte

Código da CID-10

Atenção médica (diagnóstico e tratamento precoce)

Doenças inflamatórias dos órgãos pélvicos femininos

Cólera

N70-N72

Febre tifoide e paratifoide

$\mathrm{A} 00$

Salmonella

$\mathrm{A} 01$

Shiguelose

$\mathrm{A} 02$

Outras intoxicações alimentares bacterianas

$\mathrm{A} 03$

Amebíase

A05

Infecções intestinais, decorrentes de outras agências

A06

A08

Outras infecções intestinais maldefinidas

A09

Hepatites virais

B15

Rickettsioses

A75-A79

Tripanossomíase

B56-B57

Outra doença intestinal causada por giárdia e outros protozoários

$\mathrm{A} 07$

Medidas mistas

MM1 - infecções do trato respiratório (todas as infecções respiratórias agudas, pneumonia e influenza)

MM4 - mortes violentas

Todas as lesões e intoxicações

Transtornos mentais e comportamentais decorrentes do uso de substância psicoativa

MM5 - tuberculose

MM6 - problemas de deficiências

Desnutrição

Anemias nutricionais

Outras anemias e as não especificadas

Outras doenças do sangue e dos órgãos formadores

MM7 - problemas hepáticos

Cirrose e outras doenças hepáticas crônicas

J00-J06, J10-J18, J20-J22

MM8 - doenças relacionadas com o meio ambiente

Dengue

V00-Y98

F10-F19

A15-A19

Malária

E40-E46

D50-D53

D461, D463

D731

K70-K73

Leishmaniose

Helmintíases

Toxoplasmose

A90-A91

B50-B54

B55

B65-B83

B58

Efeitos tardios do tracoma

B94

MM9 - doença pelo vírus da imunodeficiência humana (HIV)

B20-B24

MM10 - tumores relacionados com tabagismo

Tumores de laringe

C32

Tumores de traqueia

C33

Tumores de brônquios e pulmão

C34

* Código da CID-09.

CID-10: 10ạ revisão da Classificação Internacional de Doenças 32.

Nota: Foram desconsideradas as causas de morte não aplicáveis aos idosos.

Fonte: adaptado de Gómez-Arias et al. 17.

hepáticos; MM8 - doenças relacionadas com o meio ambiente; MM9 - doença pelo vírus da imunodeficiência humana (HIV); MM10 - tumores relacionados com o tabagismo.

\section{Análise estatística}

Foram elaboradas tábuas de mortalidade de múltiplo decremento, conforme o método proposto por Chiang, para o cálculo de ganhos potenciais na esperança de vida dos idosos 33 . Esse méto- 
do permite medir o impacto que cada causa de morte exerce sobre a esperança de vida em uma determinada idade, assumindo que essas causas atuem simultaneamente segundo uma determinada força de mortalidade. A tábua de mortalidade de múltiplo decremento é equivalente à tábua de mortalidade ordinária, exceto pela função de probabilidade de morte no intervalo de idade $[\mathrm{x}$ $\mathrm{x}+\mathrm{n}],{ }_{\mathrm{n}} \mathrm{q}_{\mathrm{x}}$, denominada probabilidade de mortalidades líquidas, pois são calculadas eliminandose o efeito das outras causas.

As tábuas de mortalidade de múltiplo decremento foram elaboradas excluindo-se os óbitos das causas de morte consideradas evitáveis dentro dos capítulos da CID-10 mais importantes entre os idosos: doenças do aparelho circulatório (IX), doenças do aparelho respiratório (X) e neoplasias (II). Também foram calculadas tábuas de múltiplo decremento excluindo-se os óbitos das principais causas de morte consideradas evitáveis dentro desses capítulos: doenças do coração (IX); gripe/pneumonia e bronquite, asma, enfisema (X); neoplasias da mama, do útero, da próstata e as associadas ao tabagismo (II); diabetes mellitus (IV). Os ganhos potenciais na expectativa de vida aos 60 anos, calculados por meio da comparação entre a expectativa de vida total e aquela na qual a causa de mortalidade de interesse foi omitida, foram calculados segundo o sexo.

\section{Resultados}

Na Tabela 2, é apresentado um conjunto de indicadores para as principais causas de morte (capítulos) entre idosos, incluindo as causas maldefinidas, e a participação das causas de morte evitáveis nesses capítulos. Em São Paulo, ocorreram 66.190 óbitos de idosos com até 74 anos no ano de 2007, sendo a maioria do sexo masculino $(58,8 \%)$. As principais causas de morte foram as doenças do aparelho circulatório $(36,5 \%)$ e as neoplasias (23\%). A estrutura da mortalidade proporcional de homens e mulheres é semelhante, à exceção dos óbitos por causas externas, cuja proporção é maior para o sexo masculino (Tabela 2).

Observaram-se diferenciais de gênero na intensidade da mortalidade total por causas. A taxa de mortalidade devido às doenças do aparelho circulatório, principal causa de morte para ambos os sexos, foi de 99,7 por 10 mil idosos do sexo masculino, enquanto a taxa do sexo feminino foi de 58,0 por 10 mil idosos. A taxa de mortalidade por doenças do aparelho circulatório foi $72 \%$ maior entre os homens. As causas externas e as doenças do aparelho digestivo registraram diferenças mais acentuadas no que diz respeito às razões de taxas segundo o sexo, com uma sobremortalidade masculina de 3,6 e 2,1, respectivamente.

As causas de morte evitáveis ocorridas entre idosos em São Paulo corresponderam a 62,5\% dos óbitos totais desse segmento populacional. As principais causas de morte evitáveis observadas dentro dos capítulos do aparelho circulatório, respiratório, neoplasias e endócrinas constituíram $82,6 \%$ do total de óbitos evitáveis (Tabela 3). A maior proporção de óbitos evitáveis foi observada entre as doenças do aparelho circulatório $(29,7 \%)$, que também registraram a maior taxa de mortalidade - 81,0 óbitos e 47,4 óbitos para cada 10 mil idosos do sexo masculino e feminino, respectivamente-, seguidas pelas neoplasias (Tabela 2).

Os óbitos em decorrência das principais causas de morte evitáveis corresponderam a $51,7 \%$ do total de óbitos, sendo $50,3 \%$ entre os homens e 53,6\% entre as mulheres. Contudo, a taxa de mortalidade devido às principais causas de morte evitáveis foi $60 \%$ maior entre os homens (Tabela 3 ).

As doenças do coração apresentaram a maior proporção de causas evitáveis - 29,2\% do total de óbitos ocorridos entre idosos com até 74 anos. Entre os homens, a taxa de mortalidade por doenças isquêmicas do coração, a doença mais incidente nesse grupo, foi o dobro da observada entre as mulheres (Tabela 3). Entre as doenças cerebrovasculares, a sobremortalidade masculina foi de $70 \%$, semelhante à observada para as principais causas de morte evitáveis como um todo $(1,6)$

O segundo grupo de doenças mais importante foi o das doenças do aparelho respiratório, com $9,3 \%$ do total de óbitos, sendo a taxa de mortalidade dos homens $90 \%$ maior do que a das mulheres. De fato, observa-se uma sobremortalidade masculina em todos os grupos de causas, com diferencial por sexo menos expressivo para o diabetes mellitus, cuja taxa foi $10 \%$ superior entre os homens. A sobremortalidade masculina também foi observada entre as causas específicas, sendo mais expressiva nas neoplasias malignas relacionadas ao tabagismo, com destaque para os cânceres de laringe e traqueia $(11,6)$. Somente três causas específicas apresentaram, entre os homens, taxas de mortalidade inferiores às observadas entre as mulheres: as doenças reumáticas crônicas do coração $(0,7)$, os transtornos não reumáticos da valva mitral $(0,7)$ e outras embolias e tromboses venosas $(0,5)$, sendo esta última calculada com base em valores muito pequenos.

Na Tabela 4, apresentam-se as expectativas de vida aos 60 anos $\left(\mathrm{e}_{60}\right)$ e os ganhos relativos 
Proporções de óbitos totais e evitáveis, taxas de mortalidade segundo o sexo e razão entre as taxas dos sexos masculino e feminino, pelas principais causas de morte entre idosos com até 74 anos. São Paulo, Brasil, 2007.

\begin{tabular}{|c|c|c|c|c|c|c|c|c|c|c|c|c|c|c|}
\hline \multirow[t]{3}{*}{ Capítulo da CID-10 } & \multicolumn{6}{|c|}{ Proporções } & \multicolumn{6}{|c|}{ Taxas } & \multirow{2}{*}{\multicolumn{2}{|c|}{$\begin{array}{l}\text { Razão de } \\
\text { taxas * }\end{array}$}} \\
\hline & \multicolumn{3}{|c|}{ Total de óbitos } & \multicolumn{3}{|c|}{ Óbitos evitáveis } & \multicolumn{3}{|c|}{ Total de óbitos } & \multicolumn{3}{|c|}{ Óbitos evitáveis } & & \\
\hline & $\begin{array}{l}\text { Ho- } \\
\text { mens }\end{array}$ & $\begin{array}{c}\text { Mu- } \\
\text { lheres }\end{array}$ & Total & $\begin{array}{l}\text { Ho- } \\
\text { mens }\end{array}$ & $\begin{array}{c}\text { Mu- } \\
\text { lheres }\end{array}$ & Total & $\begin{array}{l}\text { Ho- } \\
\text { mens }\end{array}$ & $\begin{array}{l}\text { Mu- } \\
\text { Iheres }\end{array}$ & Total & $\begin{array}{l}\text { Ho- } \\
\text { mens }\end{array}$ & $\begin{array}{l}\text { Mu- } \\
\text { Iheres }\end{array}$ & Total & Totais & $\begin{array}{l}\text { Evi- } \\
\text { táveis }\end{array}$ \\
\hline $\begin{array}{l}\text { I. Algumas doenças } \\
\text { infecciosas e parasitárias }\end{array}$ & 2,8 & 3,1 & 2,9 & 2,4 & 2,5 & 2,4 & 7,8 & 4,8 & 6,1 & 6,5 & 3,9 & 5,1 & 1,6 & 1,7 \\
\hline II. Neoplasias [tumores] & 22,7 & 23,3 & 23,0 & 7,1 & 8,2 & 7,5 & 62,5 & 36,8 & 48,4 & 19,4 & 13,0 & 15,9 & 1,7 & 1,5 \\
\hline $\begin{array}{l}\text { IV. Doenças endócrinas, } \\
\text { nutricionais e metabólicas }\end{array}$ & 5,2 & 7,8 & 6,2 & 4,6 & 6,7 & 5,5 & 14,2 & 12,3 & 13,2 & 12,5 & 10,7 & 11,5 & 1,2 & 1,2 \\
\hline $\begin{array}{l}\text { V. Transtornos mentais e } \\
\text { comportamentais }\end{array}$ & 0,6 & 0,3 & 0,5 & 0,5 & 0,1 & 0,3 & 4,0 & 2,9 & 3,4 & 1,3 & 0,1 & 0,7 & 1,4 & 9,0 \\
\hline $\begin{array}{l}\text { IX. Doenças do aparelho } \\
\text { circulatório }\end{array}$ & 36,3 & 36,7 & 36,5 & 29,5 & 30,0 & 29,7 & 99,7 & 58,0 & 76,8 & 81,0 & 47,4 & 62,5 & 1,7 & 1,7 \\
\hline $\begin{array}{l}\text { X. Doenças do aparelho } \\
\text { respiratório }\end{array}$ & 11,1 & 10,7 & 10,9 & 9,6 & 8,9 & 9,3 & 30,4 & 16,9 & 23,0 & 26,3 & 14,1 & 19,6 & 1,8 & 1,9 \\
\hline $\begin{array}{l}\mathrm{XI} \text {. Doenças do aparelho } \\
\text { digestivo }\end{array}$ & 6,6 & 5,5 & 6,1 & 3,8 & 3,0 & 3,5 & 18,1 & 8,6 & 12,9 & 10,4 & 4,8 & 7,3 & 2,1 & 2,2 \\
\hline $\begin{array}{l}\text { XIV. Doenças do aparelho } \\
\text { geniturinário }\end{array}$ & 1,8 & 2,2 & 2,0 & 1,2 & 1,0 & 1,1 & 5,0 & 3,4 & 4,1 & 3,2 & 1,7 & 2,4 & 1,4 & 1,9 \\
\hline $\begin{array}{l}\text { XVIII. Sintomas, sinais } \\
\text { e achados anormais } \\
\text { de exames clínicos e } \\
\text { de laboratório, não } \\
\text { classificados em outra parte }\end{array}$ & 6,4 & 5,3 & 6,0 & NA & NA & NA & 17,7 & 8,4 & 12,6 & NA & NA & NA & 2,1 & NA \\
\hline $\begin{array}{l}\text { XX. Causas externas } \\
\text { de morbidade e de } \\
\text { mortalidade }\end{array}$ & 4,1 & 2,0 & 3,2 & 4,1 & 2,0 & 3,2 & 11,3 & 3,1 & 6,8 & 11,3 & 3,1 & 6,8 & 3,6 & 3,6 \\
\hline Outras causas ** & 2,4 & 3,3 & 2,8 & 0,0 & 0,0 & 0,0 & 6,6 & 5,2 & 5,8 & 0,0 & 0,0 & 0,0 & 1,3 & 0,0 \\
\hline Total & 100,0 & 100,0 & 100,0 & 62,6 & 62,5 & 62,5 & 274,8 & 158,0 & 210,6 & 172,0 & 98,7 & 131,8 & 1,7 & 1,7 \\
\hline Total de óbitos & 38.919 & 27.271 & 66.190 & 24.361 & 17.041 & 41.402 & & & & & & & & \\
\hline
\end{tabular}

CID-10: 10ạ revisão da Classificação Internacional de Doenças 32; NA: não se aplica.

* Razão entre taxas é o quociente entre as taxas de mortalidade de homens e mulheres;

** Outras causas referem-se aos capítulos III, VI, VII, VIII, XII, XIII, XV, XVI, XVII, XIX e XXI da CID-10

Fonte: Sistema de Informações sobre Mortalidade (SIM).

obtidos por meio da eliminação de causas evitáveis, segundo o sexo. Os ganhos relativos foram calculados tendo como base as expectativas de vida do Estado de São Paulo para a população total (22,2 anos), para os homens (20,3 anos) e para as mulheres (24,0 anos). Em São Paulo, a $\mathrm{e}_{60}$ estimada por meio da eliminação das principais causas de morte evitáveis foi de 26,8 anos, sendo 25,7 anos entre os homens e 27,7 anos entre as mulheres, indicando um ganho relativo bem superior entre os homens (26,9\%), quando comparado com o das mulheres $(15,6 \%)$.

Em relação aos grupos e subgrupos de causas de morte evitáveis, observaram-se maiores ganhos na $\mathrm{e}_{60}$ para as doenças do aparelho circulatório, algo em torno de dois anos, explicados quase que totalmente pelas doenças do coração. Entre os homens, os ganhos relativos representaram $12,6 \%$ e entre as mulheres, $8,2 \%$.

A eliminação das doenças respiratórias resultaria em ganhos de $3,3 \%$, para a população total, sendo 4,3\% entre os homens e 2,5\% entre as mulheres. Esse grupo se mostrou bem homogêneo, com ganhos na expectativa de vida similares nos dois subgrupos avaliados: gripe/pneumonia e bronquite, asma, enfisema e as demais doenças das vias aéreas inferiores. 
Tabela 3

Número, proporção de óbitos, taxa de mortalidade por sexo e razão entre taxas de mortalidade dos sexos masculino e feminino, dos óbitos evitáveis decorrentes das principais doenças entre idosos de 60 a 74 anos, segundo grupos e subgrupos de causas de morte. São Paulo, Brasil, 2007.

\begin{tabular}{|c|c|c|c|c|c|c|c|c|c|c|c|}
\hline \multirow[t]{2}{*}{ Capítulo e causa da CID-10 } & \multirow[t]{2}{*}{$\begin{array}{l}\text { Tipo de } \\
\text { medida }\end{array}$} & \multicolumn{3}{|c|}{ Número } & \multicolumn{3}{|c|}{$\begin{array}{l}\text { Proporção no total } \\
\text { de óbitos }\end{array}$} & \multicolumn{3}{|c|}{ Taxa de mortalidade } & \multirow{2}{*}{$\begin{array}{l}\text { Razão } \\
\text { entre } \\
\text { taxas * }\end{array}$} \\
\hline & & Homens & $\begin{array}{l}\text { Mu- } \\
\text { Iheres }\end{array}$ & Total & Homens & $\begin{array}{l}\text { Mu- } \\
\text { Iheres }\end{array}$ & Total & Homens & $\begin{array}{c}\text { Mu- } \\
\text { lheres }\end{array}$ & Total & \\
\hline XI. Aparelho circulatório & & 11.470 & 8.173 & 19.643 & 29,5 & 30,0 & 29,7 & 81,0 & 47,4 & 62,5 & 1,7 \\
\hline Doenças do coração & & 11.367 & 7.989 & 19.356 & 29,2 & 29,3 & 29,2 & 80,3 & 46,3 & 61,6 & 1,7 \\
\hline $\begin{array}{l}\text { Doenças isquêmicas do coração } \\
\text { (I20-125) }\end{array}$ & AM & 5.586 & 3.447 & 9.033 & 14,4 & 12,6 & 13,6 & 39,4 & 20,0 & 28,7 & 2,0 \\
\hline $\begin{array}{l}\text { Doenças cerebrovasculares } \\
(160-69)\end{array}$ & AM & 3.701 & 2.713 & 6.414 & 9,5 & 9,9 & 9,7 & 26,1 & 15,7 & 20,4 & 1,7 \\
\hline Doenças hipertensivas (I10-I15) & AM & 1.178 & 1.056 & 2.234 & 3,0 & 3,9 & 3,4 & 8,3 & 6,1 & 7,1 & 1,4 \\
\hline Insuficiência cardíaca (I50) & AM & 902 & 773 & 1.675 & 2,3 & 2,8 & 2,5 & 6,4 & 4,5 & 5,3 & 1,4 \\
\hline $\begin{array}{l}\text { Doenças reumáticas crônicas do } \\
\text { coração (I05-109) }\end{array}$ & PP & 70 & 127 & 197 & 0,2 & 0,5 & 0,3 & 0,5 & 0,7 & 0,6 & 0,7 \\
\hline $\begin{array}{l}\text { Transtornos não reumáticos da } \\
\text { valva mitral (I34) }\end{array}$ & AM & 30 & 50 & 80 & 0,1 & 0,2 & 0,1 & 0,2 & 0,3 & 0,3 & 0,7 \\
\hline $\begin{array}{l}\text { Outras embolias e tromboses } \\
\text { venosas (182) }\end{array}$ & AM & 3 & 7 & 10 & 0,0 & 0,0 & 0,0 & 0,0 & 0,0 & 0,0 & 0,5 \\
\hline X. Aparelho respiratório & & 3.720 & 2.434 & 6.154 & 9,6 & 8,9 & 9,3 & 26,3 & 14,1 & 19,6 & 1,9 \\
\hline $\begin{array}{l}\text { Infecções do trato respiratório } \\
\text { (J00-J06, J10-J18, J20-J22) }\end{array}$ & MM & 1.899 & 1.279 & 3.178 & 4,9 & 4,7 & 4,8 & 13,4 & 7,4 & 10,1 & 1,8 \\
\hline Gripe e pneumonia (J10-J18) & MM & 1.898 & 1.273 & 3.171 & 4,9 & 4,7 & 4,8 & 13,4 & 7,4 & 10,1 & 1,8 \\
\hline $\begin{array}{l}\text { Bronquite, asma, enfisema e } \\
\text { outras doenças das vias aéreas } \\
\text { inferiores (J40-J47) }\end{array}$ & AM & 1.821 & 1.155 & 2.976 & 4,7 & 4,2 & 4,5 & 12,9 & 6,7 & 9,5 & 1,9 \\
\hline II. Neoplasia & & 2.747 & 2.239 & 4.986 & 7,1 & 8,2 & 7,5 & 19,4 & 13,0 & 15,9 & 1,5 \\
\hline $\begin{array}{l}\text { Tumores relacionados ao } \\
\text { tabagismo }\end{array}$ & & 1.892 & 787 & 2.679 & 4,9 & 2,9 & 4,0 & 13,4 & 4,6 & 8,5 & 2,9 \\
\hline $\begin{array}{l}\text { Neoplasia maligna dos } \\
\text { brônquios e dos pulmões (C34) }\end{array}$ & MM & 1.503 & 746 & 2.249 & 3,9 & 2,7 & 3,4 & 10,6 & 4,3 & 7,2 & 2,5 \\
\hline $\begin{array}{l}\text { Neoplasia maligna da laringe e } \\
\text { da traqueia }(\mathrm{C} 32, \mathrm{C} 33)\end{array}$ & MM & 389 & 41 & 430 & 1,0 & 0,2 & 0,6 & 2,7 & 0,2 & 1,4 & 11,6 \\
\hline Tumor maligno da mama (C50) & AM & 9 & 924 & 933 & 0,0 & 3,4 & 1,4 & 0,1 & 5,4 & 3,0 & 0,0 \\
\hline Tumor maligno da próstata (C61) & AM & 846 & NA & 846 & 2,2 & NA & 1,3 & 6,0 & NA & 2,7 & NA \\
\hline $\begin{array}{l}\text { Tumor maligno do útero } \\
\text { (C53-C55) }\end{array}$ & AM & NA & 528 & 528 & NA & 1,9 & 0,8 & NA & 3,1 & 1,7 & NA \\
\hline $\begin{array}{l}\text { IV. Endócrinas, nutricionais e } \\
\text { metabólicas }\end{array}$ & & 1.642 & 1.765 & 3.407 & 4,2 & 6,5 & 5,1 & 11,6 & 10,2 & 10,8 & 1,1 \\
\hline Diabetes mellitus (E10-14) & AM & 1.642 & 1.765 & 3.407 & 4,2 & 6,5 & 5,1 & 11,6 & 10,2 & 10,8 & 1,1 \\
\hline Total de óbitos evitáveis & & 19.579 & 14.611 & 34.190 & 50,3 & 53,6 & 51,7 & 138,2 & 84,7 & 108,8 & 1,6 \\
\hline Total de óbitos & & 38.919 & 27.271 & 66.190 & 100,0 & 100,0 & 100,0 & 274,8 & 158,0 & 210,6 & 1,7 \\
\hline
\end{tabular}

AM: atenção médica; CID-10: 10ạ revisão da Classificação Internacional de Doenças 32; MM: medidas mistas NA: não se aplica. PP: prevenção primária. * Razão entre taxas é o quociente entre as taxas de mortalidade de homens e mulheres.

Fonte: Sistema de Informações sobre Mortalidade (SIM). 
Esperança de vida e ganhos relativos na esperança de vida aos 60 anos, em relação às principais causas, segundo sexo, grupos e subgrupos de causas de morte. São Paulo, Brasil, 2007.

\begin{tabular}{|c|c|c|c|c|c|c|}
\hline \multirow[t]{2}{*}{ Causas de morte } & \multicolumn{3}{|c|}{$e_{60}$ estimado (eliminando evitáveis) } & \multicolumn{3}{|c|}{ Ganhos relativos (\%) } \\
\hline & Homens & Mulheres & Total & Homens & Mulheres & Total \\
\hline Óbitos evitáveis & 25,7 & 27,7 & 26,8 & 26,9 & 15,6 & 20,5 \\
\hline Aparelho circulatório & 22,8 & 26,0 & 24,5 & 12,6 & 8,2 & 10,2 \\
\hline Doenças do coração (I10-I15; I20-I25; 150; 160-I69) & 22,8 & 25,9 & 24,5 & 12,5 & 8,0 & 10,0 \\
\hline Outras (105-109; 134; 182) & 20,3 & 24,0 & 22,3 & 0,1 & 0,2 & 0,1 \\
\hline Aparelho respiratório & 21,1 & 24,6 & 23,0 & 4,3 & 2,5 & 3,3 \\
\hline Gripe e pneumonia (J10-J18) & 20,7 & 24,3 & 22,6 & 2,2 & 1,4 & 1,7 \\
\hline $\begin{array}{l}\text { Bronquite, asma, enfisema e outras doenças das vias aéreas } \\
\text { inferiores (J40-J47) }\end{array}$ & 20,7 & 24,3 & 22,6 & 2,1 & 1,1 & 1,6 \\
\hline Neoplasias & 20,8 & 24,5 & 22,8 & 2,8 & 1,9 & 2,3 \\
\hline Tumores relacionados ao tabagismo (C32-C34) & 20,6 & 24,2 & 22,5 & 1,8 & 0,7 & 1,2 \\
\hline Tumor maligno da mama (C50) & 20,3 & 24,2 & 22,3 & 0,0 & 0,7 & 0,4 \\
\hline Tumor maligno da próstata (C61) & 20,5 & NA & 22,3 & 1,0 & NA & 0,4 \\
\hline Tumor maligno do útero (C53-C55) & NA & 24,1 & 22,3 & NA & 0,4 & 0,2 \\
\hline Diabetes mellitus (E10-E14) & 20,6 & 24,4 & 22,6 & 1,6 & 1,6 & 1,6 \\
\hline
\end{tabular}

NA: não se aplica.

Fonte: Sistema de Informações sobre Mortalidade (SIM).

Eliminando-se os óbitos decorrentes das neoplasias, obtém-se um ganho em torno de 2,3\% na expectativa de vida, ganho inferior ao obtido para as doenças do aparelho respiratório $(3,3 \%)$ e um pouco superior ao calculado para o diabetes mellitus (1,6\%).

Os maiores diferenciais por sexo foram observados entre as neoplasias relacionadas ao tabagismo, com ganhos $167 \%$ maiores entre os homens.

\section{Discussão}

No intuito de colaborar com a redefinição das políticas e programas de saúde, novos paradigmas e indicadores têm sido propostos, já que indicadores tradicionalmente usados, como a esperança de vida, não são suficientes para o planejamento e avaliação dos programas e políticas de saúde pública 34 . Nesse sentido, é importante refletir sobre o conceito de "envelhecimento saudável" 35 e metodologia de classificação de mortes evitáveis entre idosos por doenças crônicas. Ambos têm em comum o princípio de que é preciso otimizar as oportunidades de saúde para melhorar a qualidade de vida à medida que as pessoas envelhecem.

Como assinalam Maia et al. 18, o desafio não é prolongar a vida a qualquer custo, mas, sim, dispor de adequada assistência, prevenção e promoção da saúde não somente nas idades mais tenras, mas também na fase de envelhecimento, evitando mortes prematuras de idosos. Não existe consenso quanto à idade limite para uma morte de idoso ser evitada por meio dessas ações, entretanto, há algumas décadas, tem sido proposta a extensão da idade limite da evitabilidade das causas de morte para além dos 65 anos 23,36.

Informações do Banco Mundial revelaram que as doenças crônicas (ou não transmissíveis) registraram recentemente taxas de morte prematura de cinco a nove vezes maiores do que as das doenças infectocontagiosas 37. Já está bem documentado na literatura o caráter evitável de grande parte das doenças crônicas e da possibilidade de envelhecer com qualidade de vida 1,19,38. Também há evidência acumulada sobre a iniquidade em relação à aplicação efetiva de medidas, incluindo intervenções e tratamento farmacológico, na prevenção e gestão das doenças crônicas 39 .

A metodologia de análise de causas de morte evitáveis procura mensurar o peso que essas causas têm na estrutura da mortalidade populacional e, assim, identificar as ações de prevenção, diagnóstico, tratamento precoce e adoção de tecnologias que contribuiriam para a sua redução 4 .

No presente estudo, a análise foi feita com base nas informações sobre óbitos de idosos de 
60 a 74 anos residentes no Estado de São Paulo Observou-se que o total das causas de mortes consideradas evitáveis representam $62,5 \%$ do total de óbitos ocorridos entre idosos. Essa proporção encontra-se num patamar um pouco abaixo do valor médio de $72,1 \%$ encontrado por GómezArias 40 para a Colômbia. As mortes relacionadas às principais causas de morte evitáveis, que, em grande parte, são compostas por doenças crônicas, representaram $82,6 \%$ de todos os óbitos por causas evitáveis, sendo elas as doenças do coração $(56,6 \%)$, a gripe e a pneumonia $(9,3 \%)$ e os tumores associados ao tabagismo $(7,8 \%)$.

Outro achado importante desta investigação refere-se aos potenciais ganhos em anos de vida, obtidos por meio da eliminação das principais causas consideradas evitáveis. Caso não tivessem ocorrido esses óbitos, a expectativa de vida aos 60 anos, no Estado de São Paulo, aumentaria em $20,5 \%$, passando de 22,2 anos para 26,8 anos, valor próximo ao observado para o Japão.

As desigualdades de gênero nos ganhos da expectativa de vida aos 60 anos observadas nesta pesquisa estão em consonância com os resultados obtidos por Abreu et al. 41: os homens ganhariam, proporcionalmente, mais anos de vida do que as mulheres, caso os óbitos decorrentes das principais causas de morte evitáveis fossem eliminados, $27 \%$ e $16 \%$ respectivamente. Contudo, a diferença entre as expectativas de vida de homens e mulheres seriam mantidas.

As doenças do aparelho circulatório são a principal causa de morte entre idosos. Em São Paulo, cerca de $80 \%$ das mortes desse grupo de causas são evitáveis, segundo a classificação utilizada. No Brasil, dispõe-se de políticas direcionadas para a prevenção dessas doenças. Especificamente, o Plano Nacional de Reorganização da Atenção à Hipertensão e à Diabetes Mellitus 42 visa a aumentar a prevenção, o diagnóstico, o tratamento e o controle dessas doenças por meio da reorganização da rede de atenção básica de saúde, principalmente, entre a população com 40 anos ou mais. Em consonância, a Secretaria Estadual de Saúde de São Paulo incluiu, no seu Quadro de Metas de Saúde 43, a redução das taxas de internação e mortalidade por doenças do aparelho circulatório. Entretanto, esses programas estão voltados apenas para a população com idades entre 30 e 59 anos, excluindo, assim, a população idosa.

Observou-se, ainda, neste estudo, que os ganhos relativos na esperança de vida dos idosos, caso os óbitos por diabetes mellitus não tivessem ocorrido, poderiam ser de aproximadamente $2 \%$. Embora esses ganhos sejam menores quando são comparadas outras doenças, o desenvolvimento do diabetes, sem tratamento ou controle, pode acarretar complicações mais sérias, atingindo o sistema nervoso e circulatório, consequência mais comum do diabetes. A antecipação no diagnóstico e no tratamento pode impedir tanto a morte por diabetes mellitus como por doenças do aparelho circulatório, de grande impacto na mortalidade dos idosos, como mostram os resultados 44 .

O segundo maior grupo de causas de mortes evitáveis são as doenças do aparelho respiratório, especialmente a pneumonia, principal complicação da gripe. Entre os óbitos por infecções do trato respiratório, a quase totalidade deve-se à pneumonia, sendo o risco aproximadamente duas vezes maior para os homens. Como estratégia para a redução da morbidade e da mortalidade por complicações da gripe, o Ministério da Saúde, desde 1999, incorporou à Política Nacional de Imunização 45 a vacinação de idosos contra influenza.

Pesquisas sobre diferenciais de gênero mostraram que a população masculina experimenta taxas mais elevadas de mortalidade geral e também pela maioria das causas específicas 46,47 . Este estudo corrobora esses achados, na medida em que riscos mais elevados de morte por causas evitáveis foram maiores entre os idosos do sexo masculino.

A maior sobremortalidade masculina observada entre idosos deveu-se aos tumores associados ao fumo (tumores da laringe, da traqueia, dos brônquios e pulmão), sendo a taxa de mortalidade entre os homens quase duas vezes maior do que entre as mulheres. Resultados semelhantes foram observados por Bradshaw et al. 48 , que analisaram a mortalidade por doenças crônicas na África do Sul. Esse diferencial entre os sexos pode ser explicado pela maior prevalência de homens fumantes. Dados de Curitiba (Paraná) e de Belo Horizonte (Minas Gerais) também mostraram que o impacto dos óbitos associados ao tabagismo é bem maior entre idosos do sexo masculino 49 .

As diferenças na expectativa de vida entre os sexos resultam de uma interação entre fatores biológicos, sociais e hábitos comportamentais, tornando sua explicação um processo complexo ${ }^{50}$. No entanto, a maior exposição masculina a fatores de risco, como o tabagismo, o consumo de álcool e os riscos ocupacionais, assim como a pouca utilização dos serviços de saúde 51 , é frequentemente citada como importante para o aumento nas taxas de mortalidade masculina $47,52,53$. Independentemente de terem ou não problemas de saúde, as mulheres brasileiras utilizam mais os serviços de saúde do que os homens 54 . Louvison et al. 55, com base em informações sobre idosos com idades entre 60 e 74 anos para o 
Município de São Paulo em 2000, também mostraram que as mulheres utilizam mais os serviços de saúde, de forma preventiva ou ambulatorial, reduzindo, assim, a chance de ocorrência de episódios graves e internações.

Informações recentes da pesquisa realizada pelo Ministério da Saúde (Vigilância de Fatores de Risco e Proteção para Doenças Crônicas por Inquérito Telefônico - VIGITEL) indicam que o Brasil avançou no controle do tabagismo, em face da queda expressiva na proporção de fumantes, principalmente entre os homens. Essa redução relaciona-se às medidas regulatórias, como a proibição da propaganda de tabaco e as advertências quanto aos danos causados à saúde estampados nos maços de cigarro ${ }^{56}$. A pesquisa também apontou aumento no consumo excessivo de bebidas alcoólicas, em níveis maiores para os homens. Tanto o hábito de fumar quanto o consumo de bebida alcoólica são indicadores importantes no monitoramento dos fatores de risco para doenças crônicas não transmissíveis (hipertensão arterial, diabetes e problemas cardíacos) e ambos fazem parte do comportamento masculino tradicional. Sendo assim, para sua prevenção, recomenda-se que os programas englobem aspectos culturais e comportamentais relacionados aos sexos 57 .

Segundo a Organização Mundial da Saúde (OMS) 58 , o padrão de mortalidade e morbidade entre os idosos atualmente é reflexo do comportamento vivenciado em idades mais jovens, $\mathrm{o}$ qual englobava os fatores de risco citados e acarretaram problemas crônicos na velhice. Portanto, recomenda-se que políticas de prevenção e de promoção à saúde devem ser incentivadas em idades mais jovens.

Embora a orientação de incorporar a dimensão de gênero em todas as políticas e programas para que a desigualdade diminua 59 esteja explícita no Plano de Ação Internacional para o Envelhecimento da Organização das Nações Unidas (ONU), do qual o Brasil é signatário, isso não tem se refletido nas ações de saúde desenvolvidas no País. Nas orientações do Pacto pela Saúde 60 para o biênio 2010-2011, foram definidas metas prioritárias e específicas para a implantação da Política Nacional de Atenção Integral à Saúde do Homem, todavia a política tem como públicoalvo homens entre 20 e 59 anos.

A carência de acompanhamento na atenção básica para a população idosa como um todo é reforçada pela ausência de indicadores no projeto de Monitoramento da Atenção Básica no Es- tado de São Paulo. Apesar de haver um conjunto grande de indicadores disponíveis, que já estão sendo utilizados no monitoramento, estes se limitam à idade de 59 anos.

De acordo com a classificação de GómezArias et al. 17, a prevenção e a atenção médica são medidas mais indicadas para adiar ou evitar óbitos por doenças crônicas. Estudos mostram que homens e mulheres buscam atendimento à saúde com objetivos diferentes. As mulheres utilizam os serviços de saúde como medida preventiva, tendo a possibilidade de acompanhar a evolução da doença. Já os homens utilizam mais os serviços em situações de emergência e são mais hospitalizados 61,62,63. Para modificar esse comportamento, é necessária a elaboração de campanhas, focadas na população masculina, para a conscientização da importância de exames de prevenção e de rotina.

Há duas limitações no presente estudo que merecem ser abordadas. A primeira diz respeito ao conceito de evitabilidade, definido apenas com base nas informações da causa básica, cuja identificação é mais complexa no segmento populacional de idosos, tendo em vista a elevada ocorrência de comorbidades. A segunda limitação refere-se à dificuldade em se classificar uma causa como evitável, pois nem sempre há consenso no que diz respeito à factibilidade de prevenção e ao controle de certas doenças, processo que se torna ainda mais complexo entre os idosos. O câncer colorretal foi incluído por Nolte \& McKee ${ }^{9}$ pelas evidências quanto ao seu impacto na sobrevida, enquanto Gilbert et al. 64,65 o excluiu pelas incertezas quanto às estratégias adequadas para diagnóstico precoce.

Nas listas elaboradas sobre mortalidade evitável, a maioria das intervenções adotadas nos serviços de saúde impactou nos óbitos de idosos ocorridos antes dos 75 anos. No entanto, as melhores condições de saúde, que contribuíram para o aumento na expectativa de vida, por meio de tratamento médico mais adequado, postergaram a morte para depois dos 75 anos 66. Por essas evidências, considera-se adequado estender a idade limite da morte considerada evitável 9.

Diante da real possibilidade de redução dos óbitos entre idosos por causas evitáveis e considerando o contexto epidemiológico e demográfico, recomenda-se uma investigação mais detalhada das intervenções e medidas que podem ser adotadas para evitar as mortes no Brasil, sobretudo aquelas decorrentes das doenças crônicas. 


\section{Resumen}

Las causas de muerte evitables se convirtieron en un importante indicador para evaluar los servicios de salud. Son óbitos que no deberían ocurrir ante adecuadas acciones de prevención, tratamiento, diagnóstico precoz y adopción de tecnologías apropiadas. En este artículo, se analiza el padrón y la magnitud de las causas de muerte evitables entre las principales causas de muerte de ancianos con hasta 74 años, por sexo, en el Estado de São Paulo, Brasil. Se estimó, incluso, el impacto de las causas de muerte evitables sobre la expectativa de vida de los ancianos y se observó que un 62,5\% de los óbitos entre ancianos con hasta 74 años, en São Paulo, se produjeron por esas causas. Las principales causas de muerte evitables constituyeron un $82,6 \%$ del total de óbitos evitables, destacándose la hipertensión y los tumores asociados al tabaquismo. La expectativa de vida a los 60 años aumentaría en un 20\% si esos óbitos no se produjesen. La sobremortalidad masculina fue observada en casi todas las causas de muerte evitables analizadas, lo que puede estar relacionado con una mayor exposición a factores de riesgo y a una meno utilización de los servicios de salud por los hombres. Se recomienda que las acciones de prevención y promoción de salud consideren las acentuadas diferencias de sexo y género.

Causas de Muerte; Enfermedad Crónica; Anciano;

Políticas Públicas de Salud

\section{Colaboradores}

S. Kanso participou de todas as etapas: revisão da lite ratura, definição da metodologia utilizada, processamento dos dados, análises estatísticas, interpretação, discussão dos resultados e redação final do artigo. D. E. Romero contribuiu na revisão da literatura, definição da metodologia utilizada, análises estatísticas, interpretação, discussão dos resultados e redação final do artigo. I. C. Leite colaborou na definição da metodologia utilizada, análises estatísticas, interpretação, discussão dos resultados e redação final do artigo. A. Marques contribuiu na revisão da literatura, interpretação, discussão dos resultados e redação final do artigo.

\section{Referências}

1. Sans-Sabrafen J. Entre la muerte inevitable y la búsqueda de la inmortalidad. Med Clín (Barc) 2002; 119:99-102.

2. Wilmoth J. Demography of longevity: past, present, and future trends. Exp Gerontol 2000; 35:1111-29.

3. Dempsey M. Decline in tuberculosis: the death rate fails to tell the entire story. Am Rev Tuberc 1947; 56:157-64.

4. Rutstein D, Berenberg W, Chalmers T, Child 3rd CG Fishman AP, Penin EB, et al. Measuring the quality of medical care: a clinical method. New Engl J Med 1976; 294:582-8.

5. Charlton JR, Hartley RM, Silver R, Holland WW Geographical variation in mortality from conditions amenable to medical intervention in England and Wales. Lancet 1983; 1(8326 Pt 1):691-6.
6. Holland WW. "The avoidable death" guide to Europe. Health Policy 1986; 6:115-7.

7. Taucher E. Chile, mortalidad desde 1955 a 1975 : tendencias y causas. Santiago de Chile: CELADE; 1978

8. Mackenbach J, Bouvier-Colle M, Jougla E. "Avoid able" mortality and health services: a review of aggregate data studies. J Epidemiol Community Health 1990; 44:106-11

9. Nolte E, McKee M. Does health care save lives? Avoidable mortality revisited. London: The Nuffield Trust; 2004.

10. Mackenbach J. How important have medical advances been? Improving population health in in dustrialised countries. London: Office of Health Economics; 2000. 
11. Marshall R, Keating G. Area variation of avoidable causes of death in Auckland, 1977-85. N Z Med J 1989; 102:464-5.

12. Bjerregaard P, Juel K. Avoidable deaths in Greenland 1968-1985: variations by region and period. Arctic Med Res 1990; 49:119-27.

13. Charlton J, Bauer R, Lakhani A. Outcome measures for district and regional health care planners. Commun Med 1984; 6:306-15.

14. Holland WW. European community atlas of avoidable death. Oxford: Oxford University Press; 1988.

15. Holland WW, Fitzgerald A, Hildrey S, Phillips S. Heaven can wait. J Public Health Med 1994; 16: 321-30.

16. Simonato L, Ballard T, Bellini P, Winkelmann R. Avoidable mortality in Europe 1955-1994: a plea for prevention. J Epidemiol Community Health 1998; 52:624-30.

17. Gómez-Arias R, Nolasco-Bonmatí A, PereyraZamora P, Arias-Valencía S, Rodríguez-Ospina FL, Aguirre DC. Diseño y análisis comparativo de un inventario de indicadores de mortalidad evitable adaptado a las condiciones sanitarias de Colombia. Rev Panam Salud Pública 2009; 26:385-97.

18. Maia F, Duarte Y, Lebrão M, Santos J. Fatores de risco para a mortalidade em idosos. Rev Saúde Pública 2006; 40:1049-56.

19. Kapustin J. Chronic disease prevention across the lifespan. J Nurse Pract 2010; 6:16-24

20. Wilmoth J. Search of limits between Zeus and the Salmon: the biodemography of longevity. Washington DC: Nacional Academy Press; 1997.

21. Vaupel J. Demographic analysis of aging \& longevity. Am Econ Rev 1998; 88:242-7.

22. Malta DC, Duarte EC. Causas de mortes evitáveis por ações efetivas dos serviços de saúde: uma revisão da literatura. Ciênc Saúde Coletiva 2007; 12:765-76.

23. Tobias M, Jackson G. Avoidable mortality in New Zealand, 1981-97. Aust N Z J Public Health 2001; 25:12-20.

24. Battle, RM, Pathak, D, Humble, CG, Key, CR, Vanatta, PR, and Hill, RB et al. Factors influencing discrepancies between premortem and postmortem diagnoses. JAMA 1987; 258:339-44.

25. Lloyd-Jones, DM, Martin, DO, Larson, MG, and Levy, D. Accuracy of death certificates for coding coronary heart disease as the cause of death. Ann Intern Med 1998; 129:1020-6.

26. Vasconcelos A. Estatísticas de mortalidade por causas: uma avaliação da qualidade da informação. Belo Horizonte: Associação Brasileira de Estudos Populacionais; 1996.

27. Malta DC, Duarte EC, Almeida MF, Dias MAS, Morais Neto OL, Moura L, et al. Lista de causas de mortes evitáveis por intervenções do Sistema Único de Saúde do Brasil. Epidemiol Serv Saúde 2007; $16: 233-44$

28. Verbrugge L. Gender and health: an update on hypotheses and evidence. J Health Soc Behav 1985; 26:156-82

29. World Health Organization. Global health observatory data repository. Geneva: World Health Organization; 2011.
30. Agostinho CS. Estudo sobre a mortalidade adulta, para Brasil entre 1980 e 2000 e Unidades da Federação em 2000: uma aplicação dos métodos de distribuição de mortes [Tese de Doutorado]. Belo Horizonte: Faculdade de Ciências Econômicas, UNiversidade Federal de Minas Gerais; 2009.

31. Kanso S, Romero DE, Leite IC, Moraes EN. Diferenciais geográficos, socioeconômicos e demográficos da qualidade da informação da causa básica de morte dos idosos no Brasil. Cad Saúde Pública $2011 ; 27: 1323-39$.

32. Organização Mundial da Saúde. Classificação estatística internacional de doenças e problemas relacionados à saúde, 10a revisão. v. 1. São Paulo: Centro Colaborador da OMS para a Classificação de Doenças em Português; 1995

33. Chiang C. Competing risks in mortality analysis Annu Rev Public Health 1991; 12:281-307.

34. Romero DE, Leite IC, Szwarcwald CL. Healthy life expectancy in Brazil: applying the Sullivan method. Cad Saúde Pública 2005; 21 Suppl:S7-18.

35. Organização Mundial da Saúde. Envelhecimento ativo: uma política de saúde. Brasília: Organização Pan-Americana da Saúde; 2005.

36. Mackenbach J, Kunst A, Looman C, Habbema J, van der Maas P. Regional differences in mortality from conditions amenable to medical intervention in the Netherlands: a comparison of four time periods. J Epidemiol Community Health 1988 42:325-32.

37. Adeyi O, Smith O, Robles S. Public policy and the challenge of chronic noncommunicable diseases. Washington DC: The World Bank; 2007.

38. Murray CJL, Lopez AD. The global burden of disease. Cambridge: Harvard University Press; 1996.

39. Strong K, Mathers C, Leeder S, Beaglehole R. Preventing chronic diseases: how many lives can we save? Lancet 2005; 366:1578-82.

40. Gómez-Arias RD. La mortalidad evitable como indicador de desempeño de la política sanitaria: Colombia, 1985-2001. Medellín: Universidad de Antioquia; 2006.

41. Abreu DMX, César CC, França EB. Diferenciais entre homens e mulheres na mortalidade evitável no Brasil (1983-2005). Cad Saúde Pública 2009; 25:2672-82.

42. Ministério da Saúde. Plano de reorganização da atenção à hipertensão arterial e ao diabetes mellitus no Brasil. Brasília: Ministério da Saúde; 2001.

43. Secretaria de Estado da Saúde de São Paulo. Quadro de metas de saúde. São Paulo: Estado de São Paulo; 2001.

44. Ministério da Saúde. Avaliação do plano de reorganização da atenção à hipertensão arterial e ao diabetes mellitus no Brasil. Brasília: Ministério da Saúde; 2004.

45. Ministério da Saúde. Programa nacional de imunizações. Brasília: Ministério da Saúde; 2003. (Série C. Projetos e Programas e Relatórios).

46. Trovato F, Heyen N. A varied pattern of change of the sex differential in survival in the G7 countries. J Biosoc Sci 2006; 38:391-401. 
47. Wong MD, Chung AK, Boscardin WJ, Li M, Hsieh HJ, Ettner SL, et al. The contribution of specific causes of death to sex differences in mortality. Public Health Rep 2006; 121:746-54.

48. Bradshaw D, Schneider M, Norman R, Bourne D. Mortality patterns of chronic diseases of lifestyle in South Africa. In: Steyn K, Fourie J, Temple N editors. Chronic diseases of lifestyle in South Africa. Cape Town: South African Medical Research Council; 1995. p. 5-23. (MRC Technical Report).

49. Siviero P, Myrrha L, Figoli M, Machado C. Ganhos potenciais na esperança de vida decorrentes da exclusão dos óbitos associados ao tabagismo em Curitiba e Belo Horizonte, 2003: uma aplicação do modelo de múltiplos decrementos. Belo Horizonte: Centro de Desenvolvimento e Planejamento Regional de Minas Gerais, Faculdade de Ciências Econômicas, Universidade Federal de Minas Gerais; 2010. (Texto para Discussão, 399).

50. Verbrugge L. The twain meet empirical explanations of sex differences in health and mortality. Health Soc Behav 1989; 30:282-304.

51. Westerling R. Decreasing gender differences in "avoidable" mortality in Sweden. Scand J Public Health 2003;31:342-349.

52. McCartney G, Mahmood L, Leyland A, Batty G, Hunt K. Contribution of smoking-related and alcohol-related deaths to the gender gap in mortality: evidence from 30 European countries. Tob Control 2010; 20:166-8.

53. Fiala J, Brázdová Z. A comparison between the lifestyles of men and women-parents of school age children. Cent Eur J Public Health 2000; 8:94-100.

54. Travassos C, Viacava F, Pinheiro R, Brito A. Utilização dos serviços de saúde no Brasil: gênero, características familiares e condição social. Rev Panam Salud Pública 2002; 11:365-73.

55. Louvison M, Lebrão M, Duarte Y, Laurenti R. Desigualdades nas condições de saúde e no uso de serviços entre as pessoas idosas no município de São Paulo: uma análise de gênero e renda. Saúde Coletiva 2008; 5:189-94.

56. Secretaria de Gestão Estratégica e Participativa, Secretaria de Vigilância em Saúde, Ministério da Saúde. Relatório VIGITEL 2010: Vigilância de Fatores de Risco e Proteção para Doenças Crônicas por Inquérito Telefônico. Brasília: Ministério da Saúde 2011.
57. Laurenti R, Mello-Jorge MHP, Gotlieb SLD. Perfil epidemiológico da morbi-mortalidade masculina. Ciênc Saúde Coletiva 2005; 10:35-46.

58. World Health Organization. Gender, health and ageing. Geneva: World Health Organization; 2003.

59. Organização das Nações Unidas. Plano de ação internacional para o envelhecimento. Brasília: Secretaria Especial dos Direitos Humanos; 2002.

60. Ministério da Saúde. Orientações acerca dos indicadores de monitoramento avaliação do Pacto pela Saúde, nos componentes pela vida e de gestão para o biênio 2010-2011. Brasília: Ministério da Saúde; 2011.

61. Mutran E, Ferrero K. Medical need and use of services among older men and women. J Gerontol 1988; 43:S162-71.

62. Fernandez E, Schiaffino A, Rajmil L, Badia X, Segura A. Gender inequalities in health and health care services use in Catalonia (Spain). J Epidemiol Community Health 1999; 53:218-22.

63. Dunlop DD, Manheim LM, Song J, Chang RW. Gender and ethnic/racial disparities in health care utilization among older adults. J Gerontol B Psychol Sci Soc Sci 2002; 57:S221-33

64. Gispert R, Barés M, Puigdefàbregas A. La mortalidad evitable: lista de consenso para la actualización del indicador en España. Gac Sanit 2006; 20:184-93.

65. Gispert R, Gervas J, Librero J, Bares M. Criterios para determinar la lista de causas de mortalidad evitable: una discusión inevitable. Gac Sanit 2007; 21:177-8.

66. Gispert R, Barés M, Puigdefàbregas A, Freitas A. The impact of avoidable mortality on life expectancy at birth in Spain: changes between three periods, from 1987 to 2001. J Epidemiol Community Health 2008; 62:783-9.

Recebido em 10/Ago/2012

Versão final reapresentada em 09/Nov/2012

Aprovado em 22/Nov/2012 